\title{
The correlation between MRI findings and clinical prognosis in children with purulent meningitis
}

\author{
Lei Kong \\ Beijing Children's Hospital \\ Di Li \\ Beijing Children's Hospital \\ Xin Guo \\ Beijing Children's Hospital \\ Linlin Liu \\ Beijing Children's Hospital \\ Gang Liu \\ Beijing Children's Hospital \\ Yue Liu ( $\sim$ liuyue20136@163.com )
}

Research article

Keywords: purulent meningitis; prognosis; magnetic resonance imaging

Posted Date: November 4th, 2019

DOI: https://doi.org/10.21203/rs.2.16762/v1

License: (c) (1) This work is licensed under a Creative Commons Attribution 4.0 International License. Read Full License 


\section{Abstract}

Background: To explore the correlation between magnetic resonance imaging (MRI) findings and clinical prognosis in children with purulent meningitis. Methods : Forty-nine cases of children with purulent meningitiswere collected from January 2016 to December 2018 in Beijing Children's Hospital. According to the Glasgow Outcome Scale『GOS), there were 35 patients with good prognosis and 14 patients with poor prognosis. All patients were scanned by MRI. We used Chi-square test and Logistic regression to analyse the correlation between MRI features and clinical prognosis in children with purulent meningitis. Results : Among the two groups, the poor prognosis group had the worst imaging performance, while the good prognosis group had the lighter imaging performance. Chi-square test showed that the imaging signs of cerebral edema, cerebral hemorrhage, subdural effusion, hydrocephalus, venous sinus thrombosis, cerebral hernia had statistical significance between the two groups $(P<0.05)$. Multivariate logistic regression analysis showed that the signs of cerebral edema, subdural effusion were risk factor for poor prognosis $(\mathrm{P}<0.001)$. Conclusion : There are many complications and sequelae in children with purulent meningitis. The MRI findings of cerebral edema, cerebral hemorrhage, subdural effusion, hydrocephalus, venous sinus thrombosis, cerebral hernia suggest poor prognosis. It is instructive to clinical treatment and prognosis.

\section{Background}

Purulent meningitis (PM) is also called acute bacterial meningitis, which is an acute infectious inflammation of the protective membranes covering the brain. PM is one of the most common infections of the Central Nervous System (CNS). It is characterized by fever, headache, stiff neck, nausea, vomiting, photophobia, and altered consciousness ${ }^{[1]}$. Studies have shown that the annual incidence of meningitis in the United States is 2-10/100000 ${ }^{[2]}$. The annual incidence (per 100,000 population) of probable bacterial meningitis ranged from 1.84 to 2.93 for the entire population and from 6.95 to 22.30 for children $<5$ years old in $\mathrm{China}^{[3]}$. With the improved prevention and treatment options, there was a decrease in the number of cases and mortality of patients with purulent meningitis. But it continues to be an important cause of mortality and morbidity in neonates and children ${ }^{[4,5]}$. In some instances, in spite of successful use of antibiotics, major neurologic complications occur ${ }^{[6]}$. In 2014 , British researchers found that the mortality rate of neonatal with PM dropped from $50 \%$ in 1970 to the current $10 \otimes \nabla 15 \nabla$, but the incidence rate is still 0.38 per thousand ${ }^{[7]}$. A study reported that the Glasgow coma scale score (GCS) $<8$ were independent predictors of sequelae in children with $\mathrm{PM}^{[8]}$. Pelkonen et a ${ }^{[9]}$ reported that lower GCS is a significant risk factor for predicting death or severe neurological sequelae. Therefore, early diagnosis and early treatment is very important for the prognosis of patients with PM. At present, there are few studies on the correlation between imaging findings and prognosis in neonatal PM. The relationship between imaging manifestations and prognosis of neonatal PM was studied in this paper.

MRI has better display of anatomical details of brain tissue, and its application value in PM is becoming more and more important. The complications of PM such as subdural effusion or empyema, cerebral hemorrhage, encephalitis or brain abscess, hemorrhage or venous sinus thrombosis ${ }^{[10]}$ can be directly presented by MRI. These MRI findings provide an objective basis for the prognosis of PM. This study analyzed the prognosis and imaging manifestations of 49 children with PM retrospectively, and explored the imaging signs of poor prognosis. It is important for early diagnosis and timely antibiotic treatment to improve the prognosis ${ }^{[11]}$.

\section{Methods}

\section{Subjects}

Forty-nine cases of children with purulent meningitis (mean \pm SD: $15.5 \pm 17.0$ months, range $2-89$ months; 19 female) were collected from January 2016 to December 2018 in Beijing Children's Hospital of Capital Medical University. The criteria for a definite diagnosis of bacterial meningitis included ${ }^{[12,13]}$ : (1) isolation of bacterial pathogens in one or more CSF cultures; (2) classic clinical manifestations including fever, headache, stiff neck, consciousness disturbance, and sign of meningeal irritation; (3) typical CSF findings including leukocyte counts $>0.1 \times 109 / L$ with predominant polymorphonuclear cells. All patients had been assessed by the Glasgow Outcome Scale『GOS) (Table 1), and rated as good prognosis if the GOS score was 5 , and as poor prognosis if the GOS score was 1 to 4 . By reviewing cases, we collect clinical history, symptoms, treatment options, prognosis and related complications. This study was approved by the Research Ethics Committee of the Beijing Children's Hospital affiliated to Capital Medical University.

Table 1 Glasgow Prognostic Score Scale

\begin{tabular}{l} 
Status \\
\hline Death \\
Persistent vegetative state with no significant cortical function \\
Severe disability (conscious but disabled): Because of mental or physical disability or both, patients rely on the daily support of others
\end{tabular}

Moderate disability (disability but independence): the patient's daily life is independent. Disability includes language difficulties, hemiplegia or ataxia of various degrees, as well as intellectual and memory deficits and per

Good recovery: Return to normal activity even if there is a slight neurological or p-sychological deficit 
All the patients were scanned by using a 3.0 T MR scanner (Achieva TX, Philips, the Netherlands) with an eight-channel phased-array head coil at the department of radiology of the Beijing Children's Hospital of Capital Medical University. All patients with PM were given MR scan of head, including sagittal T1-weighted imaging (T1WI), axial T1WI, T2-weighted imaging (T2WI), fluid attenuated inversion recovery (FLAIR), diffusion weighted imaging (DWI, b value = $1000 \mathrm{~S} / \mathrm{mm} 2$ ), magnetic resonance angiography (MRA), magnetic resonance venography (MRV). Contrast enhancement of gadolinium-enhanced T1-weighted imaging was performed in 20 patients. Gadolinium was injected intravenously at $0.8-2 \mathrm{ml} / \mathrm{s}$. The dosage was $0.2 \mathrm{mmol} / \mathrm{kg}$. All sequence parameters are as follows: T1Wl: repetition time $(T R)=500 \mathrm{~ms}$, echo time $(T E)=15 \mathrm{~ms}$, slice thickness $=5 \mathrm{~mm}$. T2Wl: $T R=3000 \mathrm{~ms}$, TE $=100 \mathrm{~ms}$, slice thickness $=5 \mathrm{~mm}$, T2FLAIR: TR $=7000 \mathrm{~ms}, \mathrm{TE}=150 \mathrm{~ms}$, slice thickness $=5 \mathrm{~mm}$. DWI $\mathrm{TR}=2352 \mathrm{~ms}, \mathrm{TE}=89 \mathrm{~ms}$, slice thickness $=5 \mathrm{~mm}$.

Head positioning was standardized using canthomeatal landmarks. The head was stabilized with foam pads to minimize head movements. Image with motion artifacts will be removed. Before the scan, infants and children under 7 years old needed to take chloral hydrate for sedation. The dosage was $0.5 \mathrm{ml} / \mathrm{kg}$ and the maximum dose was $10 \mathrm{ml}$.

\section{Statistical Analysis}

The statistics analysis of the data was performed by SPSS 17.0 (SPSS Inc., Chicago, IL, USA). The distribution of MRI findings between two groups were analyzed by Chi square test $(P<0.05$ suggested that the difference was significant). Factors with reference value in the univariate analysis were subjected to further Logistic regression analysis $(P<0.001$ suggested that the difference was significant).

\section{Results}

Clinical characteristics: In 49 cases of children with PM, MRI can show subdural effusion and cerebral hemorrhage, abnormal signals in basal ganglia and cerebellum, venous sinus thrombosis (Fig 1) and ventricular dilatation and cerebral hernia (Fig 2) in 14 patients with poor prognosis (female: 2, male: 12). There were no obvious abnormality was found in MRI (Fig 3 ) in 35 patients with good prognosis (female: 17, male: 18). The main clinical manifestations include headache, fever, stiff neck, nausea, vomiting.

MRI: In all 49 patients with PM, 21 cases (42.9\%) with cerebral edema (13 of the 21 patients with sequelae). 10 cases (20.4\%) with cerebral hemorrhage (9 of the 10 patients with sequelae). 11 cases (22.4\%) with encephalomalacia ( 6 of the 11 patients with sequelae). 28 cases (57.1\%) with sulcus deepening or extracerebral space widening ( 6 of the 28 patients with sequelae). 23 cases (46.9\%) with ventricular dilatation ( 6 of the 23 patients with sequelae). 8 cases (16.3\%) with hydrocephalus (all 8 patients with sequelae). 15 cases (30.6\%) with subdural effusion ( 9 of the 15 patients with sequelae): 8 of the 15 patients $(16.3 \%)$ showed frontotemporal subdural effusion, 2 of the 15 patients (4\%) showed frontoparietal subdural effusion, 5 of the 15 patients (10.2\%) showed frontotemporal subdural effusion. 8 cases (16.3\%) with venous sinus thrombosis ( all 8 patients with sequelae). 4 cases ( $8.2 \%$ ) with cerebral hernia (all 4 patients with sequelae). 16 cases (32.6\%) with mastoid-paranasal sinus mucosal thickening (14 patients with sequelae)(Table 2 ).

Relevant factors of prognosis: Chi-square test showed that the imaging signs of cerebral edema, cerebral hemorrhage, subdural effusion, hydrocephalus, venous sinus thrombosis, cerebral hernia, Mastoid-paranasal sinus mucosal thickening had statistical significance between the two groups $(P<0.05)(T a b l e ~ 3)$. Multivariate logistic regression analysis showed that the signs of cerebral edema, subdural effusion, Mastoid-paranasal sinus mucosal thickening had statistical significance $(P<0.05)($ Table 4$)$.

Table 3 Comparisons of imaging manifestations between the two groups

\begin{tabular}{|c|c|c|c|c|}
\hline Imaging findings & Good prognosis group $(n=35)$ & Poor prognosis $\operatorname{group}(\mathrm{n}=14)$ & Statistic $\left(\chi^{2}\right)$ & $\mathrm{P}$ \\
\hline cerebral edema & 8 & 13 & 20.008 & $<0.00$ \\
\hline cerebral hemorrhage & 1 & 9 & 23.231 & $<0.00$ \\
\hline Encephalomalacia & 5 & 6 & 4.689 & 0.055 \\
\hline sulcus deepening or extracerebral space widening & 22 & 6 & 1.633 & 0.222 \\
\hline ventriculomegaly & 18 & 6 & 0.294 & 0.754 \\
\hline hydrocephalus & 0 & 8 & 23.902 & $<0.00$ \\
\hline subdural effusion & 6 & 9 & 10.463 & 0.004 \\
\hline venous sinus thrombosis & 0 & 8 & 23.902 & $<0.00$ \\
\hline cerebral hernia & 0 & 4 & 10.889 & 0.005 \\
\hline Mastoid-paranasal sinus mucosal thickening & 2 & 14 & 40.425 & $<0.00$ \\
\hline
\end{tabular}

Table 4 Multivariate Logistic Regression Analysis 


\begin{tabular}{lll}
\hline Imaging findings & Score & $\mathrm{P}$ \\
\hline cerebral edema & 0.421 & 0.001 \\
\hline cerebral hemorrhage & 19.895 & 0.996 \\
\hline subdural effusion & 2.873 & $<0.001$ \\
\hline hydrocephalus & 22.966 & 0.999 \\
\hline venous sinus thrombosis & 22.966 & 0.999 \\
\hline cerebral hernia & 22.456 & 0.999 \\
\hline Mastoid-paranasal sinus mucosal thickening & 1.467 & $<0.001$ \\
\hline
\end{tabular}

\section{Discussion}

$\mathrm{PM}$ is a serious acute infection of the central nervous system (CNS). The predominant causative pathogens are Group B Streptococcus and Escherichia coli. However, mortality and morbidity remain high globally in infants ${ }^{[14-15]}$. A recent study found the incidence of bacterial meningitis in young infants to be around 0.38 per 1,000 live births and is associated with a significant case fatality rate of $8 \%[16]$. A study reported $23 \%$ of the survivors had serious CNS complications ${ }^{[17]}$. The clinical manifestations of the disease are diversified, clinical diagnosis is difficult, and the onset is rapid and progresses rapidly. The child is easily missed the best treatment opportunity ${ }^{[18]}$. In addition, sometimes blood and cerebrospinal fluid tests cannot detect pathogenic bacteria. Therefore, MRI has great application value in disease diagnosis and prognosis evaluation.

In this study, 23 of 49 PM patients had ventricular system dilatation and 15 had subdural effusion. The MRI showed ventricles system expansion, mesencephalic aqueduct dilatation and the subdural crescent T1WI high signal and T2WI high signal. Ventriculomegaly and subdural effusion are due to the subarachnoid inflammatory exudate obstructing the cerebrospinal fluid circulation pathway, resulting in hydrocephalus and high intracranial pressure, and even cerebral hernia. Once cerebral hernia occurs, the nerve tissue is seriously damaged, and the mortality rate is extremely high. Therefore, we must actively treat hydrocephalus and high intracranial pressure to prevent the occurrence of cerebral hernia. In this study, 8 patients developed venous sinus thrombosis, meningeal inflammation can extend to blood vessels, leading to vascular inflammation and thrombosis, vascular inflammation can also extend to brain parenchyma induced encephalitis, leading to subdural abscess and brain abscess. The encephalomalacia foci showed T1WI low signal and T2WI high signal in the bilateral cerebral hemisphere. This change is consistent with previous research ${ }^{[19]}$.

MRI cannot only visually display the location, extent and extent of suppurative meningitis, but also have important guiding significance for clinical treatment and prognosis. Our study showed that the probability of neurological complications in 49 patients with PM was $30 \%$, including subdural effusion (30.6\%), cerebral hemorrhage (20.4\%), hydrocephalus (16.3\%), venous sinus thrombosis (16.3\%). A study ${ }^{[20]}$ showed that in 77 cases the probability of neurological complications was $43 \%$, including subdural effusion (28.6\%), cerebral hemorrhage; encephalitis (3.9\%), hydrocephalus (2.6\%), subdural hematoma, brain abscess, subdural empyema, abscess of ventriculitis (1.3\%). A study ${ }^{[21]}$ showed that the common neurological complication were subdural effusion (47.1\%), ventriculomegaly (41.2\%), subdural empyema (21.2\%), and hydrocephalus (18.8\%). Encephalomalacia, hydrocephalus, ventriculomegaly, increased intracranial pressure were associated with a significantly higher rate of major neurological sequelae at discharge. There is consistency in these findings. These studies have shown MRI signs of hydrocephalus, subdural effusion, cerebral hemorrhage suggest a poor prognosis.

Our study found that there were differences in the formation of cerebral edema, cerebral hemorrhage, subdural effusion, hydrocephalus, venous sinus thrombosis, cerebral hernia between groups with good prognosis and those with poor prognosis. MRI manifestations of cerebral edema, subdural effusion can be used as early predictors of the final adverse outcomes, and need to be closely monitored. However, no symptoms such as brain abscess, subdural abscess and ependymitis were found in this study, which may be related to the early detection and early use of antibiotics. Patients with only meningeal lesions but no brain parenchyma have a good prognosis. Neurological complications and sequelae are common in children with purulent meningitis. Therefore, when there are signs of brain edema, cerebral hemorrhage, subdural effusion and other MRI signs, extreme treatment should be taken. Although statistics showed that the signs of mastoid-paranasal sinus mucosal thickening were statistically significant, we found that 7 of the children with mastoid-paranasal sinus mucosal thickening were younger than 3 years old and 2 were older than 3 years old. Therefore, we consider that paramastoid sinus mucosal thickening may be related to the age of the children, and can not indicate the prognosis.

\section{Conclusions}

If purulent meningitis is not treated in time, it can endanger life and cause serious central nervous system sequelae. Therefore, if the adverse prognostic signs of purulent meningitis can be evaluated at an early stage and clinical intervention at an early stage, the prognosis can be improved. However, the imaging findings suggest that prognosis is only one aspect of the study. The prognosis is related to many factors, such as clinical manifestations, biochemical indicators at admission, timely use of antibiotics, types of pathogenic bacteria, age of patients, mental state at admission, whether accompanied by shock, cytology of cerebrospinal fluid and length of stay. Because of the characteristics of the disease and condition limitation , the number of patients collected in this study is relatively small. In the follow-up study, we should increase the sample size, improve the intelligence data and reduce bias, so as to make it more instructive for clinical treatment and prognosis, and improve the quality of life of patients with purulent meningitis.

\section{Declarations}

\section{Ethics approval and consent to participate}

This study was reviewed and approved by the Ethics Committee of Beijing Children's Hospital Affiliated to Capital Medical University. Because this is a retrospectively study, we obtained the data of patients from the Medical Records and Statistics Room and we analyzed the data anonymously; thus, informed consent was not required. 


\section{Consent for publication}

Not applicable.

\section{Availability of data and materials}

The datasets during and/or analyzed during the current study are available from the corresponding author on reasonable request.

\section{Competing interests}

The authors declare that they have no competing interests.

\section{Funding}

This work was supported by the Beijing Municipal Administration of Hospitals Incubating Program (No. PX2016035) and the Beijing Health System Top Level Health Technical Personnel Training Plan (2015-3-082).

\section{Authors' contributions}

All of the authors had access to the full dataset (including the statistical reports and tables) and take responsibility for the integrity of the data and the accuracy of the data analysis. Lei Kong, Di Li, Xin Guo, Linlin Liu, Gang Liu and Yue Liu conceived and designed the study. Lei Kong, Di Li, Xin Guo and Linlin Liu collected the data and designed the analysis. Lei Kong, Di Li, and Yue Liu interpreted the data. Lei Kong and Di Li wrote the first draft of the paper. Xin Guo, Linlin Liu, Gang Liu and Yue Liu reviewed and approved the final report.

\section{Acknowledgements}

The authors would like to thank all the subjects and their families for the time and effort they dedicated to our research. Thanks to all the experts who helped complete this research. Thanks to contract grant sponsor: Beijing Municipal Administration of Hospitals Incubating Program (PX2016035) and Beijing Health System Top Level Health Technical Personnel Training Plan (2015-3-082).

\section{Authors' information (optional)}

${ }^{1}$ Department of Radiology, Beijing Children's Hospital, Capital Medical University, National Center for Children's Health, Beijing, China. ${ }^{2}$ Department of Radiology, Beijing Luhe Hospital, Capital Medical University, Beijing, China. ${ }^{3}$ Department of Infectious Disease, Beijing Children's Hospital, Capital Medical University, National Center for Children's Health, Beijing, China.

\section{References}

[1] Uiterwijk A, Koehler P J. A history of acute bacterial meningitis[J]. J Hist Neurosci. 2012, 21(3): 293-313.

[2] Viallon A, Botelho-nevers E, Zeni F. Clinical decision rules for acute bacterial meningitis: current insights [J]. Open access emergency medicine, 2016, 8: 716.

[3] Li Y, Yin Z, Shao Z, et al. Population-based surveillance for bacterial meningitis in China, September 2006-December 2009[J]. Emerg Infect Dis. 2014, 20(1): 61-69.

[4] Molyneux EM, Dube Q, Newberry L. Improving the outcome of bacterial meningitis in newborn infants in Africa: reflections on recent progress [J]. Curr Opin Infect Dis. 2015, 28(3): 215-220.

[5] Zhao Y, Liu J, Zhang J, et al. Imaging Characteristics of Purulent Meningitis Caused by Different Pathogens in Children [J]. Chin comput Med Imag. 2019, 25(2): 106-110.

[6] Jan W, Zimmerman R A, Bilaniuk L T, et al. Diffusion-weighted imaging in acute bacterial meningitis in infancy[J]. Neuroradiology. 2003, 45(9): 634-639.

[7] Okike I O, Ribeiro S, Ramsay M E, et al. Trends in bacterial, mycobacterial, and fungal meningitis in England and Wales 2004-11: an observational study[J]. Lancet Infect Dis. 2014, 14(4): 301-307.

[8] Singhi P, Bansal A, Geeta P, et al. Predictors of long term neurological outcome in bacterial meningitis[J]. Indian J Pediatr. 2007, 74(4): 369-374.

[9] Pelkonen T, Roine I, Monteiro L, et al. Risk factors for death and severe neurological sequelae in childhood bacterial meningitis in sub-Saharan Africa[J]. Clin Infect Dis. 2009, 48(8): 1107-1110.

[10] Levin S N, Lyons J L. Infections of the Nervous System[J]. Am J Med. 2018, 131(1): 25-32.

[11] Stockmann C, Spigarelli M G, Campbell S C, et al. Considerations in the pharmacologic treatment and prevention of neonatal sepsis[J]. Paediatr Drugs. 2014, 16(1): 67-81.

[12] Zhao Z, Yu JL, Zhang HB, et al. Five-year multicenter study of clinical tests of neonatal purulent meningitis [J]. Clin Pediatr (Phila),2018,57(4):389-397. 
[13] Karanika M, Vasilopoulou V A, Katsioulis A T, et al. Diagnostic clinical and laboratory findings in response to predetermining bacterial pathogen: data from the Meningitis Registry[J]. PLoS One. 2009, 4(7): e6426.

[14] Bari A. Zeeshan F, Zafar A, Ejaz H, Iftikhar A, Rathore AW. Childhood Acute Bacterial Meningitis: Clinical Spectrum, Bacteriological Profile and Outcome [J]. J Coll Physicians Surg Pak,2016,26(10):822-826.

[15] Lucas MJ, Brouwer MC, van de Beek D. Neurological sequelae of bacterial meningitis [J]. J Infect,2016,73(1):18-27.

[16] Okike I O, Johnson A P, Henderson K L, et al. Incidence, etiology, and outcome of bacterial meningitis in infants aged \&lt;90 days in the United kingdom and Republic of Ireland: prospective, enhanced, national population-based surveillance[J]. Clin Infect Dis. 2014, 59(10): e150-e157.

[17] Okike I O, Ladhani S N, Johnson A P, et al. Clinical Characteristics and Risk Factors for Poor Outcome in Infants Less Than 90 Days of Age With Bacterial Meningitis in the United Kingdom and Ireland[J]. Pediatr Infect Dis J. 2018, 37(9): 837-843.

[18] Jo J C, Yoon D H, Kim S, et al. Interim (18)F-FGD PET/CT may not predict the outcome in primary central nervous system lymphoma patients treated with sequential treatment with methotrexate and cytarabine[J]. Ann Hematol. 2017, 96(9): 1509-1515.

[19] Jaremko J L, Moon A S, Kumbla S. Patterns of complications of neonatal and infant meningitis on MRI by organism: a 10 year review[J]. Eur J Radiol. 2011, 80(3): 821-827.

[20] Namani S, Milenkovic Z, Koci B. A prospective study of risk factors for neurological complications in childhood bacterial meningitis[J]. J Pediatr (Rio J). 2013, 89(3): 256-262.

[21] Hsu M H, Hsu J F, Kuo H C, et al. Neurological Complications in Young Infants With Acute Bacterial Meningitis[J]. Front Neurol. $2018,9: 903$.

\section{Figures}
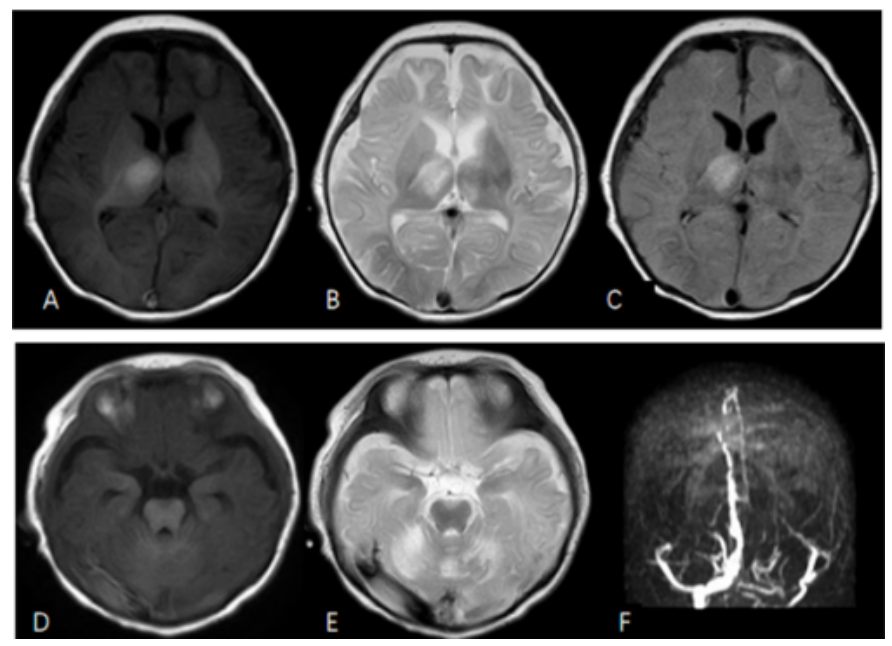

\section{Figure 1}

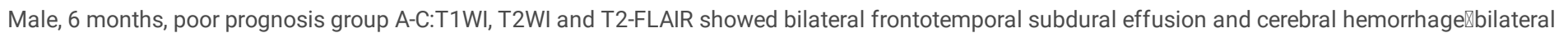
frontal cortex, basal ganglia and cerebellum with multiple T1WI high signals, T2WI low signal, FLAIR high signal囚 D-F:T1WI,T2WI,MRV showed left transverse sinus with thrombosis

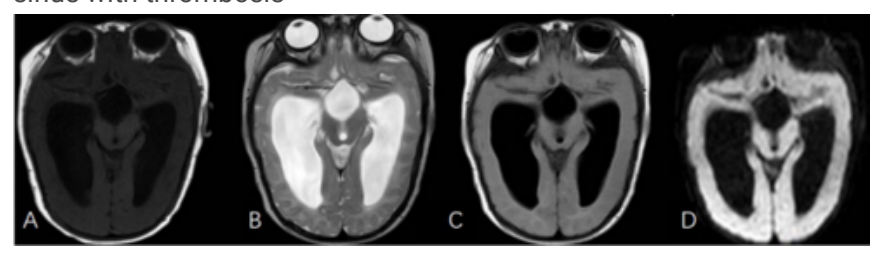

\section{Figure 2}

Male, 3 months, poor prognosis group A-D: T1WI, T2WI, T2-FLAIR, DWI showed ventricular dilatation and anterior inferior third ventricular hernia 


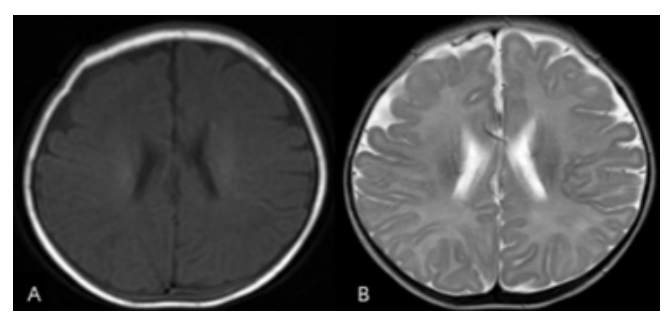

Figure 3

Female, 3 months, good prognosis group A $\mathrm{B}: \mathrm{T} 1 \mathrm{WI}, \mathrm{T} 2 \mathrm{WI}$ showed that good prognosis group had no obvious abnormal signs 